\title{
Utilização do Índice de Estilos de Aprendizagem de Felder-Soloman em Turmas de Nível Técnico, Graduação e Pós-Graduação em Computação
}

\author{
Janderson J. B. Aguiar', Joseana M. Fechine', Evandro B. Costa ${ }^{2}$ \\ ${ }^{1}$ Universidade Federal de Campina Grande (UFCG), Campina Grande - PB - Brasil \\ ${ }^{2}$ Universidade Federal de Alagoas (UFAL), Maceió - AL - Brasil \\ janderson@copin.ufcg.edu.br, joseana@dsc.ufcg.edu.br, evandro@ic.ufal.br
}

\begin{abstract}
Each individual has preferences for the way they represent and organize new information, and you can call it Learning Styles (LS). The identification of these LS can facilitate the teaching-learning process. In this paper, we present a study on the LS of computing students from different educational levels (technical, undergraduate and graduate). The extraction of these LS was carried out from the Index of Learning Styles (created by Felder-Soloman), based on the Felder-Silverman's theory. With this study, it is possible to verify the heterogeneity referring to preferences relating to the way students learn at different levels, even if they are of a specific area (computing).
\end{abstract}

Resumo. Cada indivíduo possui preferências relativas à forma com que representa e organiza novas informações, sendo possível denominar isso de Estilos de Aprendizagem (EA). A identificação desses EA pode favorecer o processo de ensino-aprendizagem. Neste artigo, é apresentado um estudo sobre os EA dos alunos de computação de diferentes níveis de ensino (técnico, graduação e pós-graduação). A extração desses EA foi realizada a partir do Índice de Estilos de Aprendizagem de Felder-Soloman, baseado na teoria de Felder-Silverman. Este estudo possibilitou verificar o grau de heterogeneidade referente às preferencias no modo de aprender dos alunos em diferentes níveis, mesmo que estes sejam de uma área específica (computação).

\section{Introdução}

O processo de aprendizagem não é vivenciado por todos os indivíduos da mesma forma, sendo desenvolvidos - como resultado da bagagem hereditária, das experiências pessoais e das exigências do ambiente - estilos de aprendizagem, que enfatizam algumas habilidades sobre as outras (KOLB, 1984).

Mesmo os estudantes de cursos específicos, como computação, apesar de geralmente terem características em comum - se comparados a estudantes de cursos de outras áreas - , ainda apresentam estilos distintos, não devendo ser tratados da mesma forma pelos facilitadores da aprendizagem (docentes, coordenadores de curso etc.).

Ao tomar consciência que cada estudante tem seus EA, os docentes tornam-se capazes de promover um ensino utilizando estratégias que promovam um aprendizado mais eficaz (FERNANDES, BENITTI e CUNHA, 2013).

Sobre as formas com que os aprendizes lidam particularmente/individualmente com as informações, o conceito de Estilos de Aprendizagem (EA) é cada vez mais 
presente em pesquisas na educação. Segundo Riding e Rayner (2000), esses estilos indicam a maneira preferida, individual e habitual com que os aprendizes organizam e representam novas informações durante o processo de aprendizagem.

Nessa temática, Coffield et al. (2004) realizaram uma revisão da literatura (utilizando os seguintes termos de busca: Learning style/s; Cognitive style/s; Conative style/s; Thinking style/s; Learning preference/s, strategy/ies, orientation/s) e identificaram 71 modelos de EA, utilizados em áreas como educação, psicologia, sociologia, entre outras. Dentre esses modelos, é possível destacar o modelo de FelderSilverman (FELDER e SILVERMAN, 1988), o mais utilizado no contexto brasileiro e internacional em pesquisas relativas à adaptação e personalização de materiais de aprendizagem (AGUIAR, FECHINE e COSTA, 2014; VALASKI, MALUCELLI e REINEHR, 2011).

Inicialmente, o modelo de Felder-Silverman foi usado por instrutores e estudantes de engenharia e ciências, sendo posteriormente aplicado em várias outras disciplinas. Para Felder e Silverman (1988), os EA referem-se aos modos pelos quais os indivíduos preferem receber e processar as informações. Ao receber, a informação externa (captada pelos sentidos) e a informação interna (que surge introspectivamente) ficam disponíveis para o indivíduo, que seleciona o material a ser processado. Ao processar, o indivíduo pode fazer uso de simples memorização ou raciocínio indutivo ou dedutivo, reflexão ou ação, introspecção ou interação com outros indivíduos. Como resultado, o conteúdo abordado no material selecionado é aprendido de uma forma ou de outra ou, então, não é aprendido.

O modelo de Felder-Silverman contempla 4 (quatro) dimensões de EA: (1) Processamento (estilos Ativo e Reflexivo); (2) Percepção (estilos Sensorial e Intuitivo); (3) Entrada (estilos Visual e Verbal); e (4) Compreensão (estilos Sequencial e Global). Inicialmente havia a dimensão Organização (estilos Indutivo e Dedutivo), removida em 2002 por Felder, defendendo que o modo indutivo obtém melhores resultados e, uma vez que os estudantes preferem o modo dedutivo, não queria que sua pesquisa servisse como justificativa para os professores continuarem a usar o modo dedutivo em suas aulas (FELDER, 2002).

Em geral, os indivíduos Ativos aprendem por meio da experimentação ativa, e compreendem as informações mais eficientemente discutindo e aplicando os conceitos; por outro lado, os Reflexivos necessitam de um tempo sozinhos para pensar/refletir sobre as informações obtidas. Os indivíduos Sensoriais preferem lidar com situações concretas, dados e experimentos; por outro lado, os Intuitivos são inovadores, gostam de lidar com abstrações, conceitos e teorias. Os indivíduos com estilo Visual aprendem mais facilmente por meio de demonstrações, diagramas, figuras, filmes, fluxogramas; por outro lado, aqueles com estilo Verbal compreendem melhor as informações transmitidas por meio de palavras. Os indivíduos Sequenciais aprendem melhor quando o conceito é expresso de maneira contínua de dificuldade e complexidade; por outro lado, os indivíduos Globais são multidisciplinares, aprendendo em grandes saltos e lidando com os conteúdos de forma aleatória (FELDER e SILVERMAN, 1988; DIAS, GASPARINI e KEMCZINSK, 2009).

Baseado nas 4 dimensões, foi desenvolvido o Índice de Estilos de Aprendizagem (Index of Learning Styles - ILS), intrumento para determinar as preferências de aprendizagem do modelo de Felder-Silverman (FELDER e SOLOMAN, 1999). 
Neste artigo é apresentada uma pesquisa realizada com variados estudantes da área de computação (no ensino técnico de nível médio, na graduação e na pósgraduação stricto sensu), com base no ILS, visando contribuir para a melhoria do processo de ensino-aprendizagem nessa área. $\mathrm{Na}$ seção 2, são comentados alguns estudos relacionados. Na seção 3, é descrita a metodologia empregada. Na seção 4, são apresentados os resultados obtidos. Na seção 5, são realizadas algumas considerações finais.

\section{Trabalhos Relacionados}

Muitos trabalhos na literatura usam teorias relacionadas à forma com que os estudantes preferem aprender.

Cury (2000) aplicou o ILS a 44 estudantes de engenharia, demonstrando que eles se apresentaram com EA preferencialmente Ativos, Sensoriais, Visuais e Sequenciais. Belhot, Freitas e Dornellas (2005) coletaram dados de 123 estudantes do curso de Engenharia de Produção Mecânica, utilizando o ILS e o inventário de Keirsey e Bates, e traçaram um perfil de EA dominantes desses estudantes.

Santos e Mognon (2010) realizaram um estudo buscando identificar os EA predominantes nos estudantes universitários. Aplicaram o ILS a 242 estudantes de diversos cursos e, dentre os resultados, indicaram que os estudantes apresentaram predominância pelos estilos Ativo, Sensorial, Visual e Sequencial.

Oliveira (2012) estudou o impacto dos EA no desempenho acadêmico do ensino de contabilidade, utilizando o ILS aplicado a 276 estudantes e 13 professores, e concluindo que é possível notar influência no desempenho dos discentes.

Fernandes, Benitti e Cunha (2013) apresentaram um levantamento feito junto a 118 estudantes da área de computação (Sistemas para Internet; Engenharia de Computação; Ciência da Computação; Tecnologia em Jogos Digitais), com base no Inventário de Estilos de Aprendizagem de Kolb, a fim de obter subsídios para melhorar o processo de ensino-aprendizagem.

Embora as pesquisas comentadas acima - e muitas outras encontradas na literatura sobre EA - sejam relacionadas à pesquisa descrita neste artigo, não foram encontrados trabalhos cujo foco tenha sido a extração (utilizando o ILS) e análise dos EA de estudantes de computação em diferentes níveis de ensino.

\section{Metodologia}

Com o objetivo de analisar semelhanças/diferenças de perfis de estudantes na área da computação, relativos aos seus EA, foi aplicado o questionário ILS a estudantes de nível técnico, graduandos e pós-graduandos (mestrandos e doutorandos) em Ciência da Computação.

O ILS abrange 44 questões objetivas (alternativa "a" ou "b"), sendo 11 para cada uma das 4 dimensões. As questões 1, 5, 9, 13, 17, 21, 25, 29, 33, 37 e 41 são relativas à dimensão Processamento, sendo a primeira alternativa (letra "a") representante do valor Ativo e a segunda (letra "b") do valor Reflexivo. As questões 2, 6, 10, 14, 18, 22, 26, $30,34,38$ e 42 referem-se à dimensão Percepção, sendo a primeira alternativa representante do valor Sensorial e a segunda alternativa representante do valor Intuitivo. 
As questões relativas à dimensão Entrada são 3, 7, 11, 15, 19, 23, 27, 31, 35, 39 e 43, sendo que a primeira alternativa representa o valor Visual e a segunda o valor Verbal. As demais questões $(4,8,12,16,20,24,28,32,36,40$ e 44) são relacionadas à dimensão Entendimento, sendo o valor Sequencial representado pela primeira alternativa e o valor Global pela segunda.

As respostas às questões do ILS fornecem duas pontuações, para cada uma das quatro dimensões, correspondentes aos dois estilos da dimensão. A diferença entre as duas pontuações indica, dentre os dois estilos, qual é o predominante/preferido pelo respondente, além de permitir conhecer a intensidade dessa preferência - pode ser leve ou quase inexistente (pontuações 1 e 3), moderada (pontuações 5 e 7) ou forte (pontuações 9 e 11) para um ou outro estilo do par da dimensão.

Os estudos de Zywno (2003), Felder e Spurlin (2005) e Litzinger et al. (2007) concordam que o ILS é um instrumento confiável, válido e adequado para identificação dos EA, embora seja recomendado que as pesquisas com tal instrumento continuem a ser realizadas.

Ao todo, foram obtidas 118 respostas, sendo 61 de estudantes de nível técnico, 36 de estudantes graduandos e 21 de estudantes pós-graduandos (mestrandos ou doutorandos).

\section{Resultados}

Inicialmente, foram analisados os dados e gerados gráficos comparativos considerando a binaridade de estilos por dimensão (Figura 1).

De maneira geral, é possível afirmar, com base na Figura 1, que os estudantes de computação tendem a ser mais Ativos, Sensoriais, Visuais e Sequenciais. Esse resultado corrobora com os obtidos em pesquisas anteriormente citadas (CURY, 2000; SANTOS e MOGNON, 2010). Entretanto, a parcela de estudantes com os estilos opostos (Reflexivos, Intuitivos, Verbais e Globais) é significativa em muitos casos. Em relação aos estudantes de graduação, por exemplo, há leve predominância do estilo Global em vez do Sequencial.

Já se percebe, com essa abordagem comparativa, a diferença entre os estudantes em todos os três níveis de ensino considerados neste estudo. Entretanto, julgou-se interessante também considerar a intensidade das preferências por estilo e, assim, os resultados foram categorizados em leve, moderado e forte (Figura 2).

Com base na Figura 2, é possível afirmar que é a minoria dos estudantes que apresenta algum estilo com o grau de intensidade forte. Em relação à dimensão Processamento, a maioria geralmente é levemente Ativa ou levemente Reflexiva. Em relação à dimensão Percepção, a maioria é levemente ou moderadamente Sensorial. Em relação à dimensão Entrada, a maioria é levemente ou moderadamente Visual. Em relação à dimensão Compreensão, embora haja predominância de ser levemente Sequencial, muitos também são levemente Globais ou moderadamente Sequenciais.

A partir da visualização gráfica dos dados relativos aos EA, considerando graus de intensidade, nota-se ainda mais como os estudantes podem ser diferentes. 


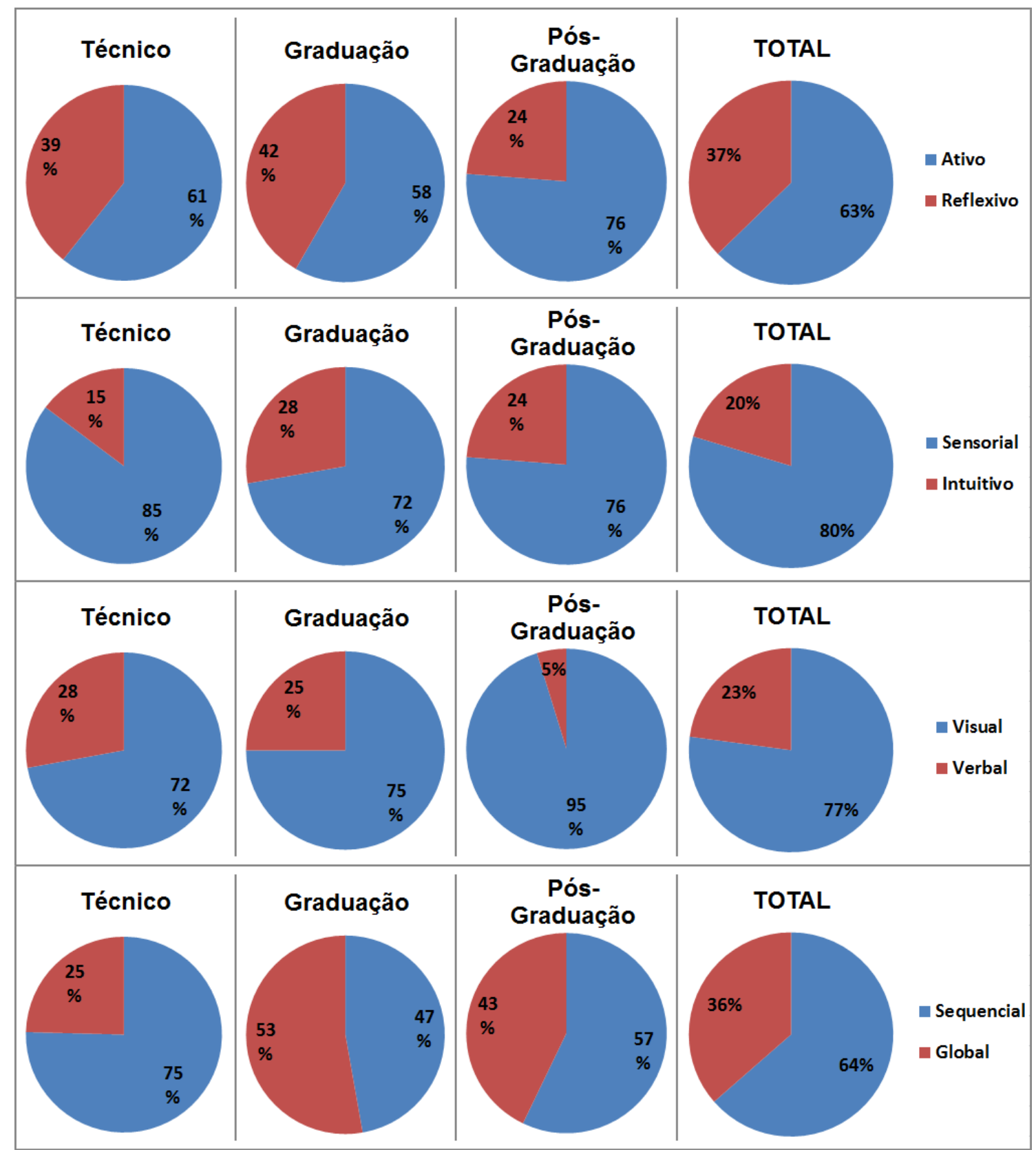

Figura 1. Comparação do resultado da aplicação do questionário ILS, em níveis diferentes de ensino de computação, considerando a binaridade dos estilos por dimensão.

Por fim, com base nos valores brutos - isto é, nem categorizados binariamente (Figura 1), nem por grau de intensidade (Figura 2) - , foi gerado o gráfico da Figura 3. No eixo horizontal, têm-se a variação para os 118 estudantes que fizeram parte desta pesquisa, respondendo o ILS. 


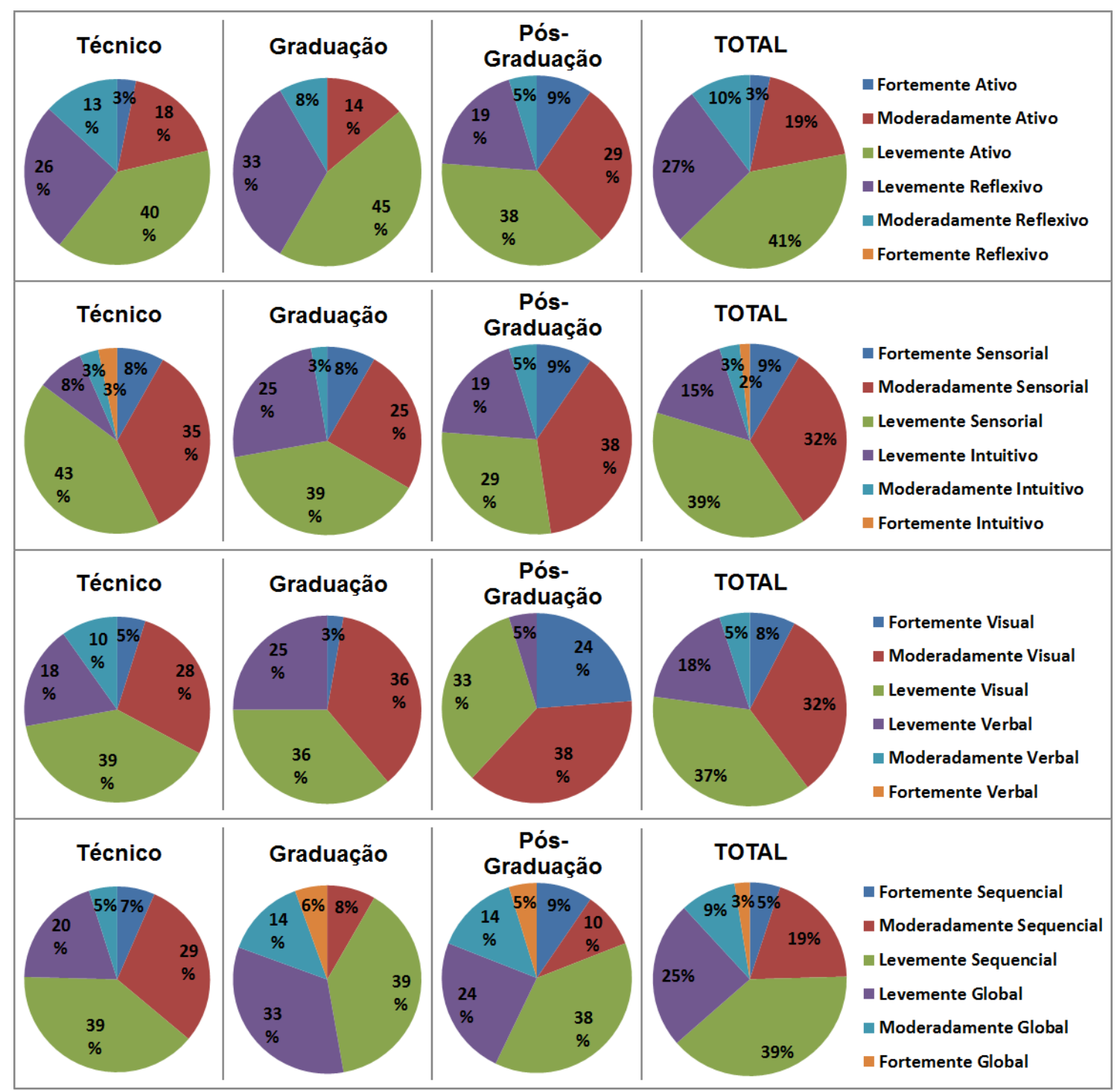

Figura 2. Comparação do resultado da aplicação do questionário ILS em níveis diferentes de ensino de computação, considerando três graus de intensidade (leve, moderado, forte) por estilo, em cada dimensão.

No eixo vertical do gráfico da Figura 3, os valores indicam a porcentagem (de 0\% a 100\%) para cada dimensão, sendo: para a dimensão Processamento, valores próximos a $0 \%$ representativos do estilo Ativo e valores próximos a $100 \%$ representativos do estilo Reflexivo; para a dimensão Processamento, valores próximos a $0 \%$ representativos do estilo Sensorial e valores próximos a $100 \%$ representativos do estilo Intuitivo; para a dimensão Processamento, valores próximos a $0 \%$ representativos do estilo Visual e valores próximos a 100\% representativos do estilo Verbal; e para a dimensão Processamento, valores próximos a $0 \%$ representativos do estilo Sequencial e valores próximos a $100 \%$ representativos do estilo Global.

Em relação aos quatro ícones coloridos da Figura 3 (que representam as dimensões de EA), é visível grande variação. Este gráfico ilustra nitidamente a heterogeneidade relativa aos EA de estudantes da área de computação e possibilita ter uma ideia das quatro dimensões combinadas por indivíduo. 


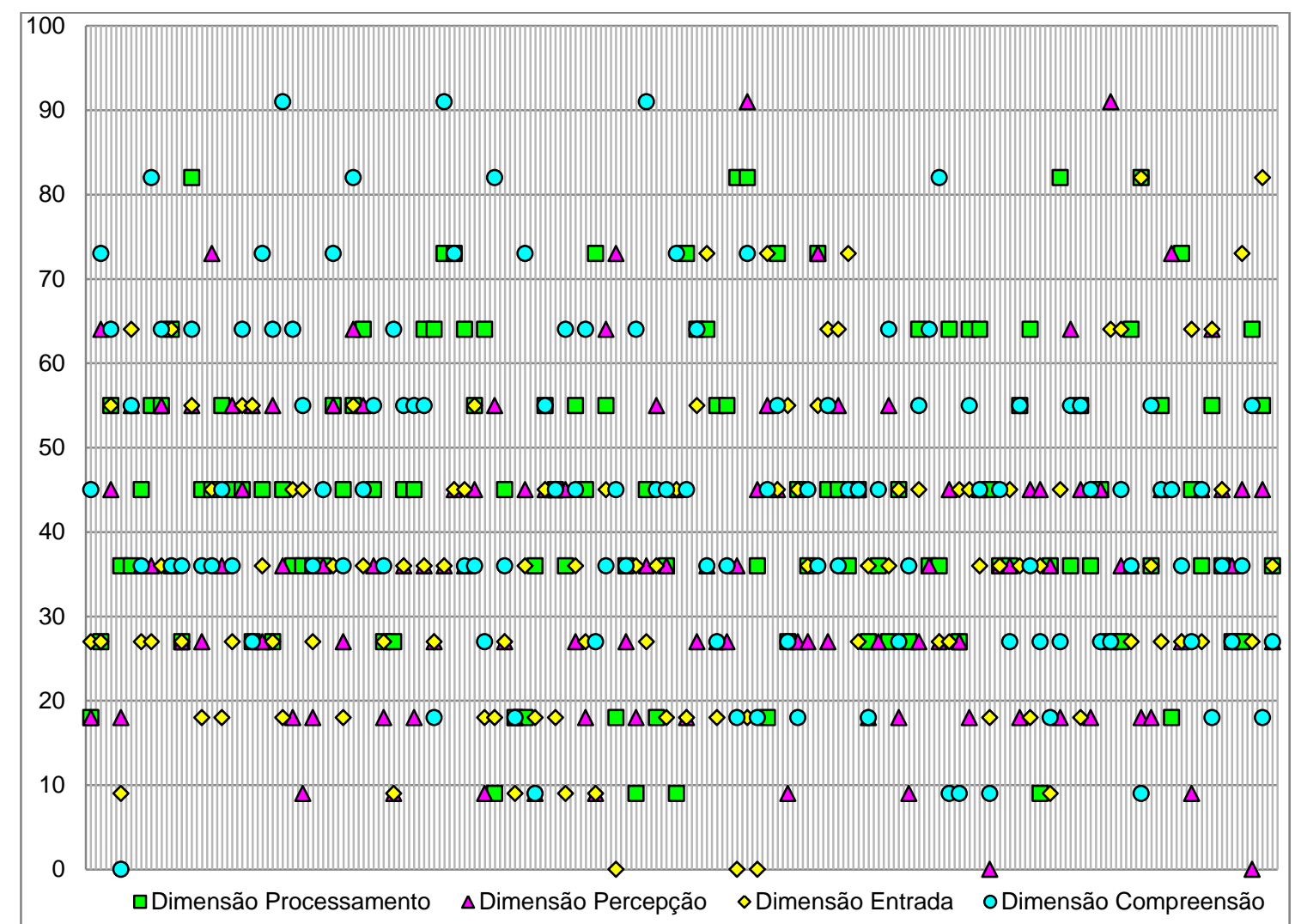

Figura 3. Ilustração da heterogeneidade dos Estilos de Aprendizagem dos 118 estudantes participantes desta pesquisa.

\section{Considerações Finais}

As teorias relacionadas a EA são essenciais para dar suporte às diferenças intrínsecas ao modo particular de aprender dos estudantes de computação. Como abordado na Seção 4, essas diferenças abrangem desde turmas de estudantes em cursos técnicos de nível médio a estudantes de programas de pós-graduação.

Além da detecção desses EA ser bastante útil, por exemplo, na personalização de ambientes em sistemas de apoio à educação na modalidade a distância, o simples uso do questionário ILS, em cursos presenciais, é um meio interessante para o professor conhecer seus estudantes e, com base nos perfis encontrados, adaptar seus planos de aula, com abordagens metodológicas e avaliativas que facilitem o processo de ensinoaprendizagem. Em relação a pós-graduandos, por exemplo, os orientadores podem fazer uso dessa teoria para sugerir pesquisas/tarefas que se adaptem aos EA de seus orientandos - e não apenas indicando temas e direções que às vezes estes pouco se interessam.

Especialmente na Educação em Computação, a criação de formulários on-line por parte dos professores não requer muitos conhecimentos além dos quais estes já possuem. Tais docentes podem facilmente automatizar o processo de geração de resultados ao implementar, na linguagem de programação que lhe convier, o algoritmo para extrair os valores dos EA com base nas respostas das 44 questões do ILS (comentado na seção 3).

Além disso, é interessante comentar que há iniciativas de uso do modelo de Felder-Silverman que não utilizam o ILS, sendo possível citar as pesquisas de Popescu, 
Trigano e Badica (2007), Graf, Kinshuk e Liu (2008) e Dorça et al. (2013). Eles sugerem a utilização de métodos baseados na análise do comportamento implícito do estudante em sistemas de aprendizagem. Graf, Kinshuk e Liu (2008) considera, dentre outras relações, que, se um estudante frequentemente visitou exercícios, há preferência por um estilo Ativo de aprendizagem. Nas pesquisas de Dorça et al. (2013) e Popescu, Trigano e Badica (2007), além da obtenção dos estilos, são realizadas atualizações dinamicamente, considerando o desempenho na avaliação de uma seção de aprendizagem (DORÇA et al., 2013) e regras como, por exemplo, um valor alto no número de postagens em fórum indica um estilo Ativo e Verbal (POPESCU, TRIGANO e BADICA, 2007).

Como apresentado por Aguiar, Fechine e Costa (2014), há um aumento de pesquisas, nos últimos anos, considerando o conceito de EA. É importante cada vez mais a utilização desse conceito para que os estudantes não sejam tratados de forma igual em relação ao modo como adquirem e produzem conhecimento.

Ao considerar um universo de alunos relativamente pequeno, a apresentação dos resultados desta pesquisa (sumarizados nas Figuras 1 e 2) não tem o objetivo de generalizar que as turmas de computação possuem determinado perfil. Esta pesquisa visou evidenciar a diferença de perfis relativos a EA de alunos de computação, mostrando a importância de considerar essas diferenças no processo de ensinoaprendizagem nessa área. Para fornecer uma generalização confiável do perfil do aluno de computação, seria necessário considerar várias instituições de ensino (de nível técnico, graduação e pós-graduação) em diferentes locais e regiões. A partir disso, poderia ser realizada, por exemplo, uma análise mais aprofundada, até mesmo com apoio de psicólogos, buscando entender como os alunos de computação, em determinado nível de ensino, possuem um estilo mais ou menos evidente em relação a alunos em outros níveis de ensino.

Almeja-se, com a divulgação desta pesquisa, motivar pesquisadores e educadores da área da computação a fazerem uso e realizarem pesquisas sobre o conceito de EA, uma vez que, como apresentado neste artigo, é válido e necessário considerar as particularidades dos estudantes dessa área independente do nível de ensino.

Como trabalho futuro, está sendo elaborado um modelo de Sistema de Recomendação Educacional baseado em EA, seguindo a teoria de Felder-Silverman, a ser avaliado por estudantes de graduação, mestrado e doutorado em Ciência da Computação.

\section{Agradecimentos}

Aos discentes participantes da pesquisa, pelas respostas ao questionário ILS, e à CAPES, pelo apoio financeiro.

\section{Referências}

AGUIAR, J. J. B.; FECHINE, J. M.; COSTA, E. (2014). Estilos Cognitivos e Estilos de Aprendizagem em Informática na Educação: um mapeamento sistemático focado no SBIE, WIE e RBIE. In: Anais do $25^{\circ}$ Simpósio Brasileiro de Informática na Educação, Dourados, p. 441-450. Disponível em: <http://www.brie.org/pub/index.php/sbie/article/view/2972/2705>. Acesso em: 15 fev. 2015. 
BELHOT, R. V.; FREITAS, A. A.; DORNELlAS, D. V. (2005). Benefícios do Conhecimento dos Estilos de Aprendizagem no Ensino de Engenharia de Produção. In: XXXIII Congresso Brasileiro de Ensino de Engenharia (COBENGE), Campina Grande. Disponível em: $<\mathrm{http}$ //www2.eesc.usp.br/aprende/images/arquivos/Beneficios_Conhecimento_Estil os_Aprendizagem_no_Ensino_Engenharia_Producao.pdf>. Acesso em: 27 abr. 2015.

COFFIELD, F.; MOSELEY, D.; HALL, E.; ECCLESTONE, K. (2004). Should we be using learning styles? What research has to say to practice. London, Learning and Skills Research Centre, Learning and Skills Development Agency. Disponível em: $<$ http://www.itslifejimbutnotasweknowit.org.uk/files/LSRC_LearningStyles.pdf $>$. Acesso em 17 fev. 2015.

CURY, H. N. (2000). Estilos de Aprendizagem de Alunos de Engenharia. In: XXVIII Congresso Brasileiro de Ensino de Engenharia (COBENGE), Ouro Preto. Disponível em: <http://www.unibarretos.com.br/v3/faculdade/imagens/nucleo-apoiodocente/ESTILOS\%20DE\%20APRENDIZAGEM\%20ALUNOS\%20ENG.pdf $>$. Acesso em: 27 abr. 2015.

DIAS, C. C. L.; GASPARINI, I.; KEMCZINSK, A. (2009). Identificação dos estilos cognitivos de aprendizagem através da interação em um Ambiente EAD. In: XXIX Congresso da Sociedade Brasileira de Computação - XVII Workshop sobre Educação em Informática (WEI), p. 489-498. Disponível em: $<$ http://www.lbd.dcc.ufmg.br/colecoes/wei/2009/011.pdf>. Acesso em: 27 abr. 2015.

DORÇA, F. A.; LIMA, L. V.; FERNANDES, M. A.; LOPES, C. R. (2013). Comparing strategies for modeling students learning styles through reinforcement learning in adaptive and intelligent educational systems: An experimental analysis. Expert Systems with Applications, v. 40, n. 6, p. 2092-2101.

FELDER, R. M. (2002). Author's Preface - June 2002. In: FELDER, R. M.; SILVERMAN, L. K. Learning and Learning and Teaching Styles in Engineering Education. Disponível em: <http://www.ncsu.edu/felder-public/Papers/LS1988.pdf $>$. Acesso em: 31 jan. 2015.

FELDER, R. M.; SILVERMAN, L. K. (1988). Learning and Teaching Styles in Engineering Education. Journal of Engineering Education, v. 78, n. 7, p. 674-681. Disponível em: $<\mathrm{http}: / / \mathrm{www} . n c s u . e d u /$ felder-public/Learning_Styles.html $>$. Acesso em: 17 fev. 2015.

FELDER, R. M.; SOLOMAN, B. A. (1999). Index of Learning Styles (ILS). Disponível em: $\quad<$ http://www4.ncsu.edu/unity/lockers/users/f/felder/public/ILSpage.html $>$. Acesso em: 15 out. 2014.

FELDER, R.; SPURLIN, J. (2005). Applications, Reliability, and Validity of the Index of Learning Styles. International Journal of Engineering Education, v. 21, p. 103112. Disponível em: $<$ http://www4.ncsu.edu/unity/lockers/users/f/felder/public/ILSdir/ILS_Validation(IJ EE).pdf $>$. Acesso em: 17 fev. 2015.

FERNANDES, A. M. R.; BENITTI, F. B. V.; CUNHA, F. S. (2013). Aplicando o Inventário de Estilos de Aprendizagem de Kolb como Ferramenta de Apoio ao Processo de Ensino Aprendizagem em Cursos de Computação. In: XXXIII Congresso 
da Sociedade Brasileira de Computação - XXI Workshop sobre Educação em Informática (WEI), p. 420-425. Disponível em: $<$ http://www.lbd.dcc.ufmg.br/colecoes/wei/2013/008.pdf>. Acesso em: 27 abr. 2015.

GRAF, S.; KINSHUK; LIU, T-C. (2008). Identifying Learning Styles in Learning Management Systems by Using Indications from Students' Behaviour. In: ICALT '08 - Eighth IEEE International Conference on Advanced Learning Technologies, p. 482-486.

KOLB, D. A. (1984). Experiential learning: experience as the source of learning and development. Englewood Cliffs: Prentice-Hall.

LITZINGER, T. A.; LEE, S. H.; WISE, J. C.; FELDER, R. M. (2007). A Psychometric Study of the Index of Learning Styles. Journal of Engineering Education, 96(4), p. 309-319. Disponível

em: $<$ http://www4.ncsu.edu/unity/lockers/users/f/felder/public/ILSdir/ILS_Validation(JE E-2007).pdf $>$. Acesso em: 17 fev. 2015.

OLIVEIRA, D. E. (2012). Impacto dos estilos de aprendizagem no desempenho acadêmico do ensino de contabilidade: uma análise dos estudantes da Universidade Federal do Rio Grande do Norte. Dissertação (Mestrado em Ciências Contábeis), Programa Multiinstitucional e Inter-Regional de Pós-Graduação em Ciências Contábeis, Universidade de Brasília, Universidade Federal da Paraíba, Universidade Federal do Rio Grande do Norte, Natal, 2012. Disponível em: $<$ http://repositorio.unb.br/handle/10482/12748>. Acesso em: 27 abr. 2015.

POPESCU, E.; TRIGANO, P.; BADICA, C. (2007). Adaptive Educational Hypermedia Systems: A Focus on Learning Styles. In: EUROCON 2007 - The International Conference on "Computer as a Tool”, p. 2473-2478.

RIDING, R.; RAYNER, S. (2000). Cognitive styles and learning Strategies understanding style differences in learning and behavior. London: David Fulton Publishers.

SANTOS, A. A. A.; MOGNON, J. F. (2010). Estilos de aprendizagem em estudantes universitários. Boletim de Psicologia, São Paulo, v. 60, n. 133. Disponível em: $<$ http://pepsic.bvsalud.org/scielo.php?script=sci_arttext\&pid=S000659432010000200009\&lng=pt\&nrm=iso>. Acesso em: 27 abr. 2015.

VALASKI, J.; MALUCELlI, A.; REINEHR, S. (2011). Revisão dos Modelos de Estilos de Aprendizagem Aplicados à Adaptação e Personalização dos Materiais de Aprendizagem. In XXII Simpósio Brasileiro de Informática na Educação - SBIE, p. 844-847. Disponível em: <http://www.brie.org/pub/index.php/sbie/article/view/1843/1605>. Acesso em: 15 out. 2014.

ZYWNO, M. S. (2003). A Contribution to Validation of Score Meaning for FelderSoloman's Index of Learning Styles". In Proceedings of the 2003 American Society for Engineering Education Annual Conference \& Exposition. Nashville, Tennessee. Disponível em: $<$ http://www4.ncsu.edu/unity/lockers/users/f/felder/public/ILSdir/Zywno_Validation _Study.pdf $>$. Acesso em: 17 fev. 2015. 